
\title{
25 Research Square \\ Influence of Pars Plana Vitrectomy on Ocular Surface using Non-Invasive Keratograph 5M
}

\section{Lingling Fan}

First Affiliated Hospital of Anhui Medical University https://orcid.org/0000-0003-1546-3748

Lun Liu

First Affiliated Hospital of Anhui Medical University

Jian Gao

First Affiliated Hospital of Anhui Medical University

Jie Zheng

First Affiliated Hospital of Anhui Medical University

\section{Yajing Xu}

First Affiliated Hospital of Anhui Medical University

Rongfeng Liao ( $\square$ liaorfayyk@126.com )

First Affiliated Hospital of Anhui Medical University

\section{Research Article}

Keywords: pars plana vitrectomy, keratograph, tear film, ocular surface, dry eye syndrome

Posted Date: December 19th, 2018

DOI: https://doi.org/10.21203/rs.2.128/v1

License: (c) (i) This work is licensed under a Creative Commons Attribution 4.0 International License.

Read Full License 


\section{Abstract}

Background: The noninvasive Keratography has been used in evaluating surgery-induced ocular surface changes, while few were known about the influence of pars plana vitrectomy (PPV) on ocular surface. This study aimed to evaluate the influence of PPV on ocular surface using Keratograph 5M.

Methods: 30 patients (30 eyes) undergoing primary PPV were recruited in the study. Ocular Surface Disease Index (OSDI) questionnaire was performed. Ocular surface parameters, including tear meniscus height (TMH), noninvasive tear break up time (NITBUT) and bulbar redness score were obtained preoperatively, at 1 week, 2 weeks, 4 weeks, 8 weeks and 12 weeks postoperatively by Keratograph $5 \mathrm{M}$. Correlations between all the clinical parameters were analyzed further.

Results: The percentages of both photophobia and gritty within 4 weeks after PPV were significantly higher than preoperation, while they decreased to the preoperative levels at both 8 weeks and 12 weeks postoperatively. The percentage of sore eyes within 2 weeks postoperatively was significantly higher than preoperation, but there were no significant differences between the percentages of preoperation and 4 weeks, 8 weeks and 12 weeks postoperatively. OSDI score increased significantly within 8 weeks postoperatively, but it returned to the preoperative level at 12 weeks. TMH was increased significantly at the first week after PPV, and recoverd to preoperative level at 2 weeks postoperatively. Both NITBUT-first and NITBUT-average shortened significantly within 8 weeks postoperatively, but they gradually improved to the preoperative levels at 12 weeks. Bulbar redness score was significantly higher than preoperative level within 4 weeks postoperatively, but it returned to the preoperative level at 8 weeks. NITBUT-first and NITBUT-average had a significant positive correlation at each visit. OSDI score had a significant positive correlation with bulbar redness, and TMH had a significant positive correlation with NITBUT-average at both 1 week and 2 weeks postoperatively.

Conclusions: Keratograph $5 \mathrm{M}$ can provide a reliable noninvasive method to assess the influence of PPV on ocular surface. PPV may cause various changes in both symptoms and signs of ocular surface damages at an early stage, while all these changes will return to preoperative levels gradually at 12 weeks postoperatively.

\section{Background}

Pars plana vitrectomy (PPV) was first introduced as a new treatment for vitreoretinal diseases by Machemer Robert in 1971. With the evolution and development of vitrectomy machines and related instruments, surgical techniques have been improved and the the application of PPV has increased significantly in recent years. However, many patients undergoing PPV have complained of dry eye related irritative symptoms postoperatively.

Dry eye syndrome is a multifactorial disease of tears and ocular surface due to tear deficiency and over evaporation, which can be caused by many complex factors, including inflammation, hyperosmolarity of tears, and neurosensory abnormalities, as well as unstable tear films[1,2]. It leads to ocular discomfort 
and visual impairment, which can impact the quality of life greatly [3]. Bulbar redness, or conjunctival hyperaemia, is a non-specific ocular response due to multiple ocular surface disorders, including conjunctivitis, anterior eye inflammation, contact lens wear and dry eye syndrome. There are also many reports about the influence of surgical interventions on ocular surface, such as corneal refractive surgery[4], cataract surgery[5] and glaucoma surgery[6]. Evaluation of tear film and bulbar redness may help us to know the surgery-induced ocular surface changes and guide appropriate treatments. Nevertheless, the influence of PPV on ocular surface is poorly understood so far.

Traditional methods evaluating dry eye have been widely used in clinical practice, including Schirmer test and fluorescein tear breakup time (TBUT). However, these invasive examinations are subjective, difficult and time-consuming, which need patients to cooperate very well. Additionally, these invasive examinations have been shown to have poor accuracy and reproducibility. The noninvasive Keratography $5 \mathrm{M}$, advanced Placido topograph, provides reliable objective measurements of ocular surface parameters including tear meniscus height (TMH), noninvasive TBUT (NITBUT) and bulbar redness score, with acceptable repeatability and reproducibility $[7,8]$. It has been used in evaluation of ocular surface in recent years, especially the diagnosis of dry eye.

In this study, we used the Keratograph $5 \mathrm{M}$ (Oculus Optikgeräte $\mathrm{GmbH}$, Wetzlar, Germany) to evaluated the changes of ocular surface after PPV, which is beneficial to manage PPV induced ocular surface damages.

\section{Methods}

\section{Participants}

This prospective, descriptive study was approved by the Ethics Committee of The First Hospital Affiliated of Anhui Medical University, and followed the tenets of the Declaration of Helsinki. Written informed consent was acquired from every patient. From April 2018 to October 2018, a total of 30 eyes from 30 patients who underwent primary 23-gauge PPV in our hospital were recruited. Their age ranged from 43囚 75 years with a mean of $59.33 \pm 7.68$ years. Half of them were male and the other half were female. All the patients were followed up for 3 months. Exclusion criteria were: 1 ) age $\leq 18$ or $\geq 75$ years; 2 ) any eye diseases influencing ocular surface (e.g. keratoconjunctivitis, ectropion, hypophasis or glucoma); 3 ) history of ocular injury or other ocular surgery within 6 months; 4) use of topical ocular medications influencing ocular surface within one week before the operation; 5 ) any other drugs used for postoperative complications during the follow-up period; 6 ) the follow-up period less than 3 months.

\section{Surgical Techniques}

The three port conventional 23-gauge PPV was done in all the 30 eyes by a single vitreoretinal surgeon under retrobulbar anesthesia induced with $0.1 \%$ Ropivacaine Hydrochloride. During the surgery, blood, anterior-posterior traction, fibrovascular tissues and membranes were removed as completely and safely as possible. For the patients with macular hole, internal limiting membranes stained with indocyanine green were peeled to at least one disc diameter from the edge of the hole. Endolaser photocoagulation or 
retinal cryotherapy was done for sealing retinal hole. Filtered air or silicone oil was used as intraocular tamponade if necessary. After removal of the cannula from the ports, the 8.0 sutures were put on the sclera and then it was checked for leakage.

After surgery, all the patients with intraocular air or silicone oil tamponade were instructed to maintain a prone position to achieve better effects. Additionally, all patients were given topical eye drops of tobramycin-dexamethasone (TobraDex, Alcon) and pranoprofen (Pranopulin, Senju) for four times daily for 1 month, and tropicamide-phenylephrine (Mydrin-P, Santen ) was used twice daily for 2 weeks.

\section{Ocular Surface Disease Index (OSDI) questionnaire for ocular symptoms}

Subjective symptoms of ocular irritation were assessed by the 12-item OSID questionaire. We modified the questionnaire by deleting the 4 and 5 items, which assess the existence of blurred and poor vision. Because it was difficult to distinguish the change of these symptoms caused by PPV alone or combined with PPV induced dry eye condition. OSDI score was calculated by the following formula:Total points of all answered questions $\times 100 /$ Total number of answered questions $\times 4$.

\section{Ketography 5M}

Clinical examinations were all performed by the same ophthalmic technician using the Keratograph $5 \mathrm{M}$ in accordance with the manufacturer's instructions. All the ocular surface parameters were obtained preoperatively, at 1 week, 2 weeks, 4 weeks, 8 weeks and 12 weeks postoperatively. Additionally, all examinations were performed sequentially with 5 minutes break as follows:

TMH: The subjects were instructed to look at a central target in the system and blink normally. The keratograph was set to "Tear film Scan (TF-Scan), tear meniscus" mode to capture an image of the ocular surface focused on the tear film following the manufacturer's instructions as previously reported[9]. The central $\mathrm{MH}$ was measured manually by cursors provided by the system.

NITBUT: Tear film stability was assessed in "TF-Scan, NITBUT"mode. After manual focusing, the patients were asked to blink twice and then keep their eyes open as long as possible. At the same time, a video was recorded showing the presentation of tear film breakup over time. The NITBUT-first and NITBUTaverage were generated automatically. The simultaneous time of the tear film starts to breakup was the NITBUT-first. NITBUT-average indicated the mean of all TBUTs occurring on the whole observed cornea.

Bulbar redness: Bulbar redness was measured in "R-Scan"mode. Subjects were instructed to focus on the light target. Then image of exposed bulbar conjunctiva was captured manually and the redness score was generated automatically. The score was calculated based on the area percentage ratio between blood vessels and the rest of the analyzed bulbar conjunctiva.

\section{Statistical Analysis}


Statistical analysis was performed by SPSS 19.0 software. All values were expressed by mean \pm standard deviation (SD). The Kolmogorov-Smirnov test was applied to test the normality of the data. One-way analysis of variance and paired $t$ test were used for comparing differences in all variables. Correlations between all variables were tested using the Pearson's correlation test. $P$ values less than 0.05 were considered statistically significant.

\section{Results}

\section{Ocular surface symptoms and OSDI score}

Most patients complained of ocular surface discomfort after PPV, mainly including photophobia, gritty and sore eyes. Distributions of these symptoms during the follow-up were shown in Figure 1A. The percentages of either photophobia or gritty increased significantly at 1 week, 2 weeks and 4 weeks postoperatively $(P<0.001, P<0.001, P=0.003 ; P<0.001, P<0.001, P=0.001$, respectively), while they decreased to the preoperative levels at both 8 weeks and 12 weeks postoperatively $(P=0.347, P=0.228$; $P=0.08, P=0.448$, respectively). The percentage of sore eyes at either 1 week or 2 weeks postoperatively was significantly higher than preoperation $(P<0.001, P=0.011$, respectively), but there were no significant differences between the percentages of preoperation and 4 weeks, 8 weeks and 12 weeks postoperatively ( $P=0.161, P=0.161, P=0.554$, respectively).

Table 1 showed OSDI score at each visit and the change of OSDI score was shown in Figure 1B. Compared with the preoperative value, the OSDI scores were all significantly lower at 1 week, 2 weeks, 4 weeks and 8 weeks postoperatively $(P<0.001, P<0.001, P<0.001, P=0.024$, respectively). While, there was no significant difference between the OSDI scores of preoperation and 12 weeks postoperatively $(P=1)$.

Table 1 Clinical parameters of preoperation and each visit postoperatively (mean+SD, $\mathrm{n}=30$ )

\begin{tabular}{lllllll}
\hline Clinical parameters & Baseline & 1 week & 2 weeks & 4 weeks & $\mathbf{8}$ weeks & 12 weeks \\
\hline OSDI score & $2.3 \pm 3.44$ & $25.69 \pm 12.74$ & $19.62 \pm 11.57$ & $11.39 \pm 8.12$ & $4.64 \pm 4.81$ & $2.36 \pm 3.82$ \\
TMH (mm) & $0.29 \pm 0.11$ & $0.34 \pm 0.13$ & $0.31 \pm 0.1$ & $0.29 \pm 0.12$ & $0.28 \pm 0.08$ & $0.26 \pm 0.08$ \\
NITBUT-first (s) & $12.11 \pm 3.28$ & $4.58 \pm 1.7$ & $6.52 \pm 2.58$ & $8.86 \pm 2.82$ & $10.65 \pm 2.8$ & $12.46 \pm 3.11$ \\
NITBUT-average (s) & $19.09 \pm 2.77$ & $8.63 \pm 1.94$ & $14.4 \pm 2.8$ & $11.72 \pm 2.73$ & $16.83 \pm 3.05$ & $19.15 \pm 2.86$ \\
Bulbar redness score & $1.66 \pm 0.5$ & $2.69 \pm 0.74$ & $2.24 \pm 0.67$ & $1.98 \pm 0.65$ & $1.71 \pm 0.47$ & $1.57 \pm 0.43$ \\
\hline
\end{tabular}

OSDI: Ocular Surface Disease Index, TMH: tear meniscus height, NITBUT: noninvasive tear breakup time 


\section{Ocular surface parameters measured by Keratograph 5M}

Table 1 summarized the ocular surface parameters at each visit, including TMH, NITBUT-first, NITBUTaverage and bulbar redness score.

In comparasion with preoperative $\mathrm{TMH}$, the value was significantly lower at 1 week postoperatively $(P=0.012)$, while the values at 2 weeks, 4 weeks, 8 weeks and 12 weeks postoperatively were not significantly different from preoperation ( $P=0.336, P=0.665, P=0.508, P=0.088$, respectively). The change in $\mathrm{TMH}$ was shown in Figure 2A.

The change in NITBUT-first was shown in Figure 2B. NITBUT-first values at 1 week, 2 weeks, 4 weeks and 8 weeks postoperatively were all significantly lower than preoperation $(P<0.001, P<0.001, P<0.001$, $P<0.001$, respectively). However, there was no significant difference between the values at 12 weeks postoperatively and preoperation $(P=0.172)$.

Similar to the changes in NITBUT-first and OSDI score, NITBUT-average was significantly shortened after PPV, but gradually improved as time passed (Figure 2C). The values at 1 week, 2 weeks, 4 weeks and 8 weeks postoperatively were significantly lower than preoperation $(P<0.001, P<0.001, P<0.001, P<0.001$, respectively). However, the values were not significantly different between 12 weeks postoperatively and preoperation $(P=0.781)$.

The change in bulbar redness score was shown in Figure 2D. The score was significantly higher at 1 week, 2 weeks and 4 weeks postoperatively than preoperation ( $P<0.001, P<0.001, P<0.001$, respectively). However, compared to preoperation, there were no significant differences at both 8 weeks and 12 weeks postoperatively $(P=0.184, P=0.108$, respectively).

\section{Correlations between clinical parameters}

Significant positive correlation was found between NITBUT-first and NITBUT-average at each visit ( $\mathrm{r}=0.548, P=0.002 ; \mathrm{r}=0.586, P=0.001 ; \mathrm{r}=0.58, P=0.001 ; \mathrm{r}=0.587, P<0.001 ; \mathrm{r}=0.634, P=0.001 ; \mathrm{r}=0.584$, $P=0.001$; respectively). In addition to this, we also found that OSDI score had a significant positive correlation with bulbar redness ( $\mathrm{r}=0.393, P=0.031 ; \mathrm{r}=0.46, P=0.011$; respectively), and TMH had a significant positive correlation with NITBUT-average ( $\mathrm{r}=0.49, P=0.006 ; \mathrm{r}=0.421, P=0.021$; respectively) at both 1 week and 2 weeks postoperatively.

\section{Discussion}

Compared with other traditional methods, Oculus Keratograph is an noninvasive and objective method to assess ocular surface, which is more likely to be applied in clinical practice with high practicability. Many studies reported that the stability of ocular surface was damaged after some surgery, causing dry eye 
related symptoms and signs, such as photorefractive keratectomy, laser in situ keratomileusis and cataract surgery. In this study, we analyzed the changes of ocular surface after PPV via OSDI questionnaire, and measuring TMH, NITBUT and bulbar redness via keratograph 5M.

In the current study, we found many patients complained of eye discomfort, especially photophobia, gritty and sore eyes, which relieved at 8 weeks postoperatively. OSDI questionnaire was used to assess symptoms of ocular surface subjectively in our study, which is one of the most commonly used questionnaires to assess dry eye. Michael et al. reported that OSDI questionnaire had superior discriminative ability in assessing dry eye signs compared with the McMonnies Dry Eye Questionnaire scores, Standard Patient Evaluation of Eye Dryness and 5-item Dry Eye Questionnaire[10]. Our results showed that OSDI score increased significantly at 1 week, 2 weeks, 4 weeks and 8 weeks after PPV, and it returned to preoperative level at 12 weeks postoperatively. This changing pattern corresponds with our clinical observation that few patients complain of eye discomfort after 8 weeks postoperatively.

Schirmer test is a traditional method to detecting tear secretion, while it's an invasive operation which induces reflex tearing. A positive correlation between $\mathrm{TMH}$ and Scherimer test value has been reported [11]. Thus, TMH can be considered as a noninvasive test for tear secretion and it's also a sensitive indicator of tear deficiency before dry eye appearance [12]. Our study showed TMH increased significantly within the first week after PPV, and it recovered to preoperative level afterwards. PPV induced stimultation of ocular surface may contribute to reflex tearing, such as surgical incisions, sutures and bulbar conjunctival edema. Along with the incision healing, sutures absorption, conjunctival edema subsiding and trauma repair, tear secretion decreases and returns to the baseline level.

TBUT reflects the stability of tear film, which is a important index to evaluate dry eye [13]. Fluorescein TBUT is widely used in clinical examination, while the fluorescein dye can destabilize the tear film and it has been reported that fluorescein BUT can be affected by the volume of fluorescein. Oculus Keratograph can detect the very early changes of tear film and record very low NITBUT objectively with high reliability and repeatability $[14,15]$. Stephanie et al. reported that NITBUT measured by Keratograph 4 can be substituted for the numerical fluorescein TBUT value [16]. The present study recorded the changes of both NITBUT-first and NITBUT-average after PPV via Keratograph 5M. We found both of them were decreased significantly within 8 weeks postoperatively, especially at the first week, and recovered to the basline levels gradually at 12 weeks postoperatively. Similar to our study, Servet et al. reported that fluorescein TBUT impaired up to 1 month after phacoemulsification, followed by a recovering trend toward the preoperative level at 12 weeks [17]. There was a significant positive correlation between NITBUT-first and NITBUT-average at each visit, demonstrating either of them can be used to evaluate tear film stability.

Golbet cells within conjunctival epithelium are special cells secreting mucins onto ocular surface [18]. Mucins secreted plays an important role in maintaining tear film stability, which can assist with removal of debris from the tear film and contribute to the hydrophilicity of tear film [19-21]. Previous studies reported that the density of goblet cells didn't return to preoperative level even at 3 months after cataract 
surgery [22], but MUC5AC could recover to the preoperative level at 3month after phacotrabeculectomy [23]. Heimann et al. reported that there was a significant decrease in MUC5AC positive goblet cells and distribution change of MUC1 in the conjunctival specimens of patients undergoing posterior segment surgery [24], which may contribute to the development of tear film destability and dry eye. The decrease in golbet cells has been reported to be correlated with operative time of cataract surgery [25]. Compared with cataract and glucoma surgery, the operative time of PPV is longer, which may contribute to the decrease in goblet cells and tear film destability.

Operation induced damage to the cornea and conjunctiva destabilizes tear film. Intraoperative ocular surface manipulation, vigorous irrigation and microscopic light exposure were reported to be related with dry eye after cataract surgery [26], which may be associated with reduced globet cell density. Thus, we presumed that ocular surface damage during PPV contributed to tear film destability. Additionally, surgical incisions, conjunctival sutures and edema lead to morphological changes of ocular surface, which may also affect tear distribution and leading to decrease of tear film stability [27]. In this study, we found there were positive correlations between TMH and NITBUT-average at both 1 week and 2 weeks after PPV, demonstrating that surgery-induced reflex tearing also aggravates tear film destability.

Benzalkonium chloride is the most common preservative used in eye drops. It can cause lots of ocular adverse effects, including dry eye, ocular inflammation, trabecular meshwork degeneration and even optic nerve injury, which is associated with its mitotoxic effect [28]. In our study, all the three eye drops used postoperatively contained benzalkonium chloride which may also contribute to tear instability and dry eye postoperatively.

Bulbar redness is a common clinical sign after ophthalmic surgery, and it may reflect the response of ocular surface to pathogenic stimuli and severity of inflammation. Fujita et al. reported that IL-1 $\beta$, IL-6 and IL-8 increased significantly after PPV [29]. Thus, inflammatory events of ocular surface can be evaluated via detecting bulbar redness. In clinical practice, bulbar redness is mainly assessed by photographic scales, including Institute for Eye Research scale, the Efron scale, and the Validated Bulbar Redness grading scale [30]. Unlike the image-based scales, keratograph is the first commercially available device which can evaluate bulbar redness via analyzing the captured images automatically and objectively. Wu et al., reported that its reproducibility was much higher than other three image-based scales for evaluating bulbar redness [31]. In our study, the bulbar redness score increased significantly within the 4 weeks postoperatively, but it recovered to the preoperative level at 8 weeks postoperatively. We found there were also significant correlations between OSDI score and bulbar redness at both 1 week and 2 weeks after PPV. These results correspond with our clinical observation that most patients complain eye discomfort within the 4 weeks after PPV, especially within the 2 weeks postoperatively.

However, the sample size of this study is very small, which needs to be expanded. In addition to these, PPV associated risk factors will be analyzed further to reduce ocular surface damage.

\section{Conclusion}


In conclusion, to the best of our knowledge, this is the first study to evaluate ocular surface after PPV via noninvasive keratograph, including tear film and bulbar redness. Our results demonstrated that PPV caused various changes of the symptoms and signs of ocular surface damage at an early stage, while these changes returned to preoperative levels gradually afterwards. This study can provide guidance for further treatment of ocular surface after PPV.

\section{Declarations}

\section{Ethics approval and consent to participate}

The study was approved by the Ethics Committee of The First Hospital Affiliated of Anhui Medical University, and followed the tenets of the Declaration of Helsinki. Written informed consent was acquired from every patient.

\section{Consent for publication}

Not applicable.

\section{Availability of data and material}

The datasets generated and/or analyzed during the current study are not publicly available due to the further research in progress, but are available from the corresponding author on reasonable request.

\section{Competing interests}

All the authors declare no competing interest.

\section{Funding}

This study was supported by the grant from The First Hospital Affiliated of Anhui Medical University (2017kj26). The sponsor had no role in the design of the study and collection, analysis, and interpretation of data and in writing the manuscript.

\section{Authors' contributions}

FLL, LL and LRF contributed to the conception of research idea, study design and supervision, data analysis and interpretation. FLL was a major contributor in writing the manuscript. GJ, ZJ and XYJ contributed to data collection and analysis. LRF gave the final approval of manuscript to publish. All authors approve the final manuscript.

\section{Acknowledgements}

Not applicable. 


\section{References}

1. Shimazaki J. Definition and Diagnostic Criteria of Dry Eye Disease: Historical Overview and Future Directions. Investigative ophthalmology \& visual science. 2018; 59(14): DES7-DES12.

2. Yamaguchi T. Inflammatory Response in Dry Eye. Investigative ophthalmology \& visual science. 2018; 59(14): DES192-DES199.

3. Shiraishi A, Sakane Y. Assessment of Dry Eye Symptoms: Current Trends and Issues of Dry Eye Questionnaires in Japan. Investigative ophthalmology \& visual science. 2018; 59(14): DES23-DES28.

4. Bower KS, Sia RK, Ryan DS, Mines MJ, Dartt DA.Chronic dry eye in photorefractive keratectomy and laser in situ keratomileusis: Manifestations, incidence, and predictive factors. Journal of cataract and refractive surgery. $2015 ; 41(12): 2624-2634$.

5. Sutu C, Fukuoka H, Afshari NA. Mechanisms and management of dry eye in cataract surgery patients. Current opinion in ophthalmology. 2016; 27(1): 24-30.

6. Zhong S, Zhou H, Chen X, Zhang W, Yi L. Influence of glaucoma surgery on the ocular surface using oculus keratograph. International ophthalmology. 2018; doi: 10.1007/s10792-018-0869-3.

7. Cox SM, Nichols KK, Nichols JJ. Agreement between Automated and Traditional Measures of Tear Film Breakup.Optometry and vision science: official publication of the American Academy of Optometry. 2015; 92(9): e257-263.

8. Koh S, Ikeda C, Watanabe S, Oie Y, Soma T, Watanabe H, et al. Effect of non-invasive tear stability assessment on tear meniscus height. Acta ophthalmologica. 2015; 93(2): e135-139.

9. Arriola-Villalobos P, Fernández-Vigo Jl, Díaz-Valle D, Peraza-Nieves JE, Fernández-Pérez C, Benítez-DelCastillo JM. Assessment of lower tear meniscus measurements obtained with Keratograph and agreement with Fourier-domain optical-coherence tomography. The British journal of ophthalmology. 2015; 99(8): 1120-1125.

10. Wang MTM., Xue AL, Craig JP. Comparative Evaluation of 5 Validated Symptom Questionnaires as Screening Instruments for Dry Eye Disease. JAMA Ophthalmol. 2018; doi:

10.1001/jamaophthalmol.2018.5243.

11. Altan-Yaycioglu R, Sizmaz S, Canan H, Coban-Karatas M. Optical coherence tomography for measuring the tear film meniscus: correlation with schirmer test and tear-film breakup time. Curr Eye Res. 2013; 38(7):736-742.

12. Xie W, Zhang D, Chen J, Liu J, Yu Y, Hu L. Tear menisci after laser in situ keratomileusis with mechanical microkeratome and femtosecond laser. Investigative ophthalmology \& visual science. 2014; 55(9): 5806-5812. 
13. Tsubota K. Short Tear Film Breakup Time-Type Dry Eye. Investigative ophthalmology \& visual science. 2018; 59(14): DES64-DES70.

14. Best N, Drury L, Wolffsohn JS. Clinical evaluation of the Oculus Keratograph. Cont Lens Anterior Eye. 2012; 35(4): 171-174.

15. Tian L, Qu JH, Zhang XY, Sun XG. Repeatability and Reproducibility of Noninvasive Keratograph 5M Measurements in Patients with Dry Eye Disease. J Ophthalmol. 2016; 2016: 8013621.

16. Cox SM, Nichols KK, Nichols JJ. Agreement between Automated and Traditional Measures of Tear Film Breakup. Optom Vis Sci. 2015; 92(9): e257-263.

17. Cetinkaya S, Mestan E, Acir NO, Cetinkaya YF, Dadaci Z, Yener HI. The course of dry eye after phacoemulsification surgery. BMC ophthalmology. 2015; 15: 68.

18. Gipson IK. Goblet cells of the conjunctiva: A review of recent findings. Prog Retin Eye Res. 2016; 54 : 49-63.

19. Ablamowicz AF, Nichols JJ. Ocular Surface Membrane-Associated Mucins.

Ocul Surf. 2016; 14(3):331-341.

20. Baudouin C, Rolando M, Benitez Del Castillo JM, Messmer EM, Figueiredo FC, Irkec M, et al. Reconsidering the central role of mucins in dry eye and ocular surface diseases. Progress in retinal and eye research.2018; pii: S1350-9462(18)30062-4.

21. Hori Y. Secreted Mucins on the Ocular Surface. Hori Y. Investigative ophthalmology \& visual science. 2018; 59(14): DES151-DES156.

22. Oh T, Jung Y, Chang D, Kim J, Kim H. Changes in the tear film and ocular surface after cataract surgery. Japanese journal of ophthalmology. 2012; 56(2): 113-118.

23. Liu W, Li H, Lu D, Liang J, Xing X, Liu A, et al. The tear fluid mucin 5AC change of primary angleclosure glaucoma patients after short-term medications and phacotrabeculectomy. Molecular vision. 2010; 16: 2342-2346.

24. Heimann H, Coupland SE, Gochman R, Hellmich M, Foerster MH. Alterations in expression of mucin, tenascin-c and syndecan-1 in the conjunctiva following retinal surgery and plaque radiotherapy. Graefe's archive for clinical and experimental ophthalmology. 2001; 239(7): 488-495.

25. Cho YK, Kim MS. Dry eye after cataract surgery and associated intraoperative risk factors. Korean J Ophthalmol. 2009; 23(2): 65-73.

26. Li XM, Hu L, Hu J, Wang W. Investigation of dry eye disease and analysis of the pathogenic factors in patients after cataract surgery. Cornea. 2007; 26(9 Suppl 1):S16-20. 
27. Xie W. Recent advances in laser in situ keratomileusis-associated dry eye. Clinical \& experimental optometry. 2016; 99(2): 107-112.

28. Datta S, Baudouin C, Brignole-Baudouin F, Denoyer A, Cortopassi GA. The Eye Drop Preservative Benzalkonium Chloride Potently Induces Mitochondrial Dysfunction and Preferentially Affects LHON Mutant Cells.Investigative ophthalmology \& visual science. 2017; 58(4):2406-2412.

29. Fujita A, Uchino E, Otsuka H, Arimura N, Noda Y, Ishibashi T, et al. Ocular surface molecule after transconjunctival vitrectomy. The British journal of ophthalmology.2011; 95(3): 419-423.

30. Macchi I, Bunya VY, Massaro-Giordano M, Stone RA, Maguire MG, Zheng Y, et al. A new scale for the assessment of conjunctival bulbar redness. The ocular surface. 2018; 16(4):436-440.

31. Wu S, Hong J, Tian L, Cui X, Sun X, Xu J. Assessment of Bulbar Redness with a Newly Developed Keratograph. Optometry and vision science. 2015; 92(8): 892-899.

\section{Figures}
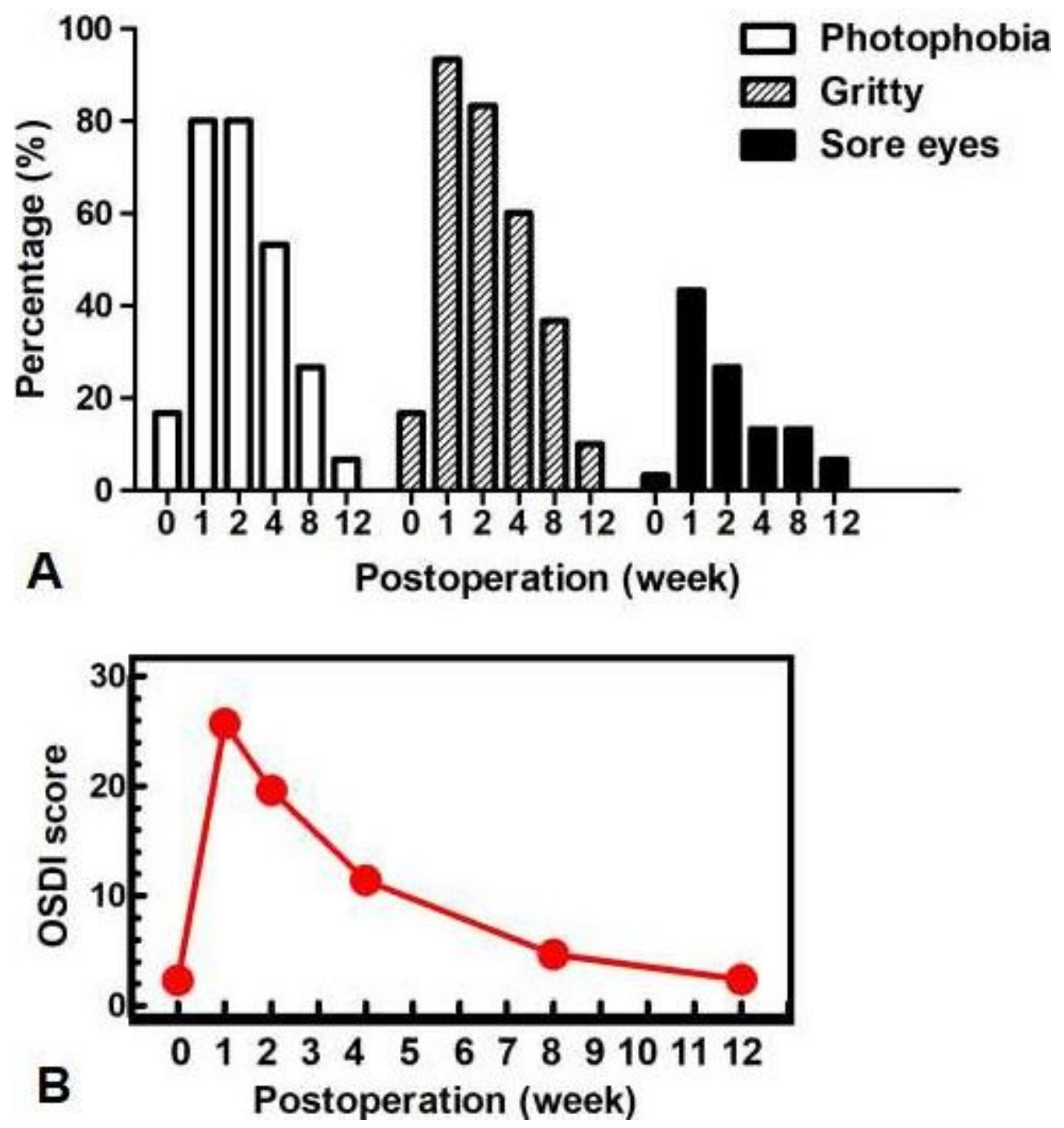
Figure 1

Distribution of ocular surface symptoms and change in OSDI score. A: Distribution of photophobia, gritty and sore eyes; $\mathrm{B}$ : Change in OSCl score.


Figure 2

Changes in ocular surface parameters after vitrectomy. A: Changes in TMH; B: Changes in NITBUT-first; $\mathrm{C}$ : Changes in NITBUT-average; D: Changes in Bulbar redness. 